\title{
6 A new instrumentalism?
}

\author{
Haidy Geismar
}

This chapter explores the relationship between digital anthropology and material culture studies, using the 'digital' as a useful tool to unravel the entanglements between theory, method, and the production of value around social research, both inside and outside of the university. The movement of digital anthropology from university-based research contexts into other, often corporate, research environments (where it is often transformed from digital anthropology into digital ethnography) is a good place to explore the realworld consequences of academic theories about the relations between things and concepts, structure and form, media and sociality. If Miller and HaapioKirk, ask in this volume how anthropology can matter and to whom, here I explore some of the analytic and interpretive tensions surrounding the ways in which digital anthropology and digital ethnography are entangled when they move from academia to become objects in the world.

The conflation of subject and object, theory and practice, form and content, which has been explored within the broad church of material culture studies (MCS), might be seen to find its apotheosis within the digital, which is simultaneously constituted as a context or subject for social research, as a method (such as digital ethnography or data visualisation), and as an object within social research (for instance big data or social media). I am caught here between celebrating the emergence of a specifically 'digital anthropology' (as articulated by Miller and Horst 2012) and the simultaneous need for critical engagement with the problems raised by the conflation of the digital as both object of study and analytic category (see Rode 2011). Rather than fixate on definitions of the digital here (see Geismar 2012, 2018), my focus in this chapter is to explore the fundamental tension between approaches that locate the digital as an object in the world (like denim jeans or a work of art), versus those that see the digital as a structuring concept (like media or materiality).

MCS is sometimes also described as a 'material turn' in reference to the range of different disciplinary and national approaches that are united by a shared interest in the material within social theory, and in the world. There are distinct threads within the turn. 'Thing Theory' emerged from literary criticism to explore narrative from the perspective of the material world (e.g. Brown 2001; Lamb 2011). 'Actor Network Theory' emerged within sociology 
and science and technology studies and has significantly worked to demolish ontological distinctions between subjects and objects by exploring the ways in which knowledge is produced within networked assemblages of diverse actants (e.g. Latour 1991). Anthropology's material turn is often recognised as a return to earlier preoccupations with 'material culture' that historically were formative for the discipline (Hicks and Beaudry 2010). Within anthropology, MCS therefore signifies both a renewed interest in the specific contexts and materiality of artefacts, but also recognises the made and material world as a fundamental component of human experience.

A further strand within the material turn is new materialism, 'a term ascribed to a range of contemporary perspectives in the arts, humanities and social sciences that have in common a theoretical and practical "turn to matter" (Fox and Alldred 2018: 1). The work of the 'new' here signals a perceived departure from Marxist inflected political critique of 'old materialism,' with its primary focus on modes and means of production and the social structures these produce. If old materialisms argue that objects are lightning rods for the production of class, inequalities and human values, new materialisms seek to move beyond the determining factor of the human, arguing for an analytic approach that understands matter and materials as a priori, in worlds that may not be dominated by human processes of making and signification (see Ingold 2007).

Accompanying the new materialism is a parallel turn from an 'old instrumentalism' in which academic work was in the service of knowledge production in a variety of applied contexts (from the formation of cultural knowledges to assist with the process of colonial governance through to understanding of the other side in war) towards a 'new instrumentalism' of market-driven utility, which Miller and Haapio-Kirk also discuss in this volume. ${ }^{1}$ We might link how some strands of new materialism reject the founding humanism of the social sciences and humanities, to the emergence of a new instrumentalism that rejects an 'old' notion of academic instrumentalism of critique in favour of 'impact': value that can be measured in relation to a baseline of some kind of number (citations, hits, downloads, numbers of viewers/ listeners, grant income, and so on; see Collini 2018). Digital media has been a lightning rod for the emergence of this new knowledge economy within the university, for instance in the emergence of digital humanities which, some of its critics declare, 'has played a leading role in the corporatist restructuring of the humanities $[, \ldots]$ pushing the discipline toward post-interpretative, non-suspicious, technocratic, conservative, managerial, lab-based practice' (Allington et al. 2016). Critics of Digital Humanities see this focus on technical problems and machine-based learning as a direct refusal of critical thinking, and also as fundamentally apolitical, chafing against critical enquiry by promoting a 'post-critical,' machine-generated positivism (but see Risam 2009 for an articulation of post-colonial digital humanities).

I argue here that these converging strands of new materialism and new instrumentalism find their apotheosis within many mainstream understandings 
of the digital, producing a kind of leviathan within MCS and, indeed, within anthropology more broadly. For instance, the shift towards seeing the material world as transcendent matter beyond the human is mirrored in prevailing popular discourses of the digital that interpret data as an objective view of the world, often obscuring the ways that algorithms and data sets internalise cultural values (see Crawford and Paglan 2019). In other instances, the emergence of software, or platform, studies naturalises a corporate infrastructure as the universe that structures activity both inside and outside platforms (see Boellstorff 2008). The inability, often due to lack of research access, to fully account for corporate infrastructures of digital platforms implicitly reorients the participatory register for research in ways similar to those documented by Marilyn Strathern in her account of how the choices around making family through new reproductive technologies naturalise consumer capitalism in determining analytic as well as political subjectivities around modern kinship (Strathern 1992). Within this dystopian digital landscape anthropology is often positioned as a form of critical engagement and a place to evidence alternative practices and experiences from within. However, it remains the case that the digital is increasingly naturalised as both a research tool and object of study, without enough attention to this broader contextual dynamic. In the rest of this chapter, I unpack some of the emergent tensions within digital anthropology that highlight ongoing concerns within MCS regarding the political implications of our theories of things. I then trace how these emerge, albeit often implicitly, within the nascent methodology of digital ethnography. Finally, focusing on a student project by way of example, I highlight the need for a more applied critical examination of the nature of the digital itself.

\section{From material culture to digital anthropology}

The Digital Anthropology programme was established in 2009 in the Department of Anthropology at University College London (UCL) by Daniel Miller as an explicit continuation of several theoretical perspectives he had already been developing for many years under the rubric of MCS. This new programme marked out a trajectory very different to other emergent fields of enquiry into the digital, which at that time were still largely preoccupied with questions of the virtual and the immaterial (see, e.g. Boellstorff 2008), and which rarely positioned themselves in the explicit context of material culture. In a manifesto of sorts, Miller and Horst (2012) positioned the digital as formally integral to the production of self and society in diverse cultural contexts. Viewing the expressive qualities of digital media within a Hegelian process of producing subjectivity or identity, they defined the digital as a form of mediation through practices of consumption and self-making, building on Miller's early work that had focused on the generative capacities and social resonance of mass-produced commodities (1987). Miller and Horst listed six core themes of digital anthropology, all of which emerge from their earlier 
ethnographic work on material culture. They articulated commitments to holism, cultural relativism, and to exploring how objects are able to activate dialectics of normativity within particular social worlds. Miller and Horst's vision of digital anthropology qua MCS was therefore not one particularly interested in the qualities of the digital as an object per se, but one focused on the ways in which digital media could be seen to refract broader cultural and social worlds and identities, and indeed help us to better understand them. ${ }^{2}$

Miller's earlier work on material culture moved away from the prevailing interpretive frames for objects of semiotics, symbolism, and signification, in which objects were illustrative of deeper concepts that structured social organisation. It also moved away from the tradition of Marxist approaches to the study of objects through processes of production, arguing for a focus on consumption and circulation, and for attention to the ongoing social life of things (cf. Appadurai 1986b). Over a number of studies, and in relation to a large array of different case studies (from denim jeans to Coca-Cola, saris to interior design), Miller and his co-authors have argued that objects and, most importantly, ubiquitous everyday things, played an active (rather than illustrative) role in processes of subjectification (e.g. Miller 1987; Banerjee and Miller 2003; Miller and Woodward 2012). In the same way, Miller and Horst's initial definition of digital technology broke away from several strands of contemporary media theory, responding to detractors of digital media, who present it as a radical break with past media traditions (e.g. Turkle 2012) to argue that digital technologies are fundamentally normative, mediating no more or less than any other cultural expression or communication. They argued, 'one of the major contributions of a digital anthropology would be the degree to which it finally explodes the illusions we retain of a non-mediated, noncultural, predigital world' (2012: 12; see also Coupaye, Chapter 4).

Horst and Miller's definition of digital anthropology also responded to debates within MCS privileging what might be called a classic version of social anthropology over a broad clustering of perspectives that could be described either as post-humanist, or broadly technologically determinist in their orientation towards the material and digital world (see, for instance, Whitehead and Wesch 2012). For instance, in the multi-sited research project, Why We Post, also discussed in his contribution (with Haapio-Kirk) to this volume, Miller and his co-researchers have argued that Facebook (or QQ in China) could be understood as distinct within the particular local landscapes of, for example, Industrial China (Wang 2016) or south-east Turkey (Costa 2016), where culturally and socially defined norms were brought into social media. One of the primary findings of Why We Post was that 'it's the people who use social media who create it, not the developers of platforms. ${ }^{3}$

This approach to digital anthropology is quite different to contemporaneous studies of the digital in other fields, which tend to upscale the form and structure of the digital from metaphor to structuring reality and often sublimate the social into a growing commitment to a more top-down technologically and materially led enquiry. Examples of this can be found in influential 
treatises on the digital, such as Bratton's formulation of The Stack (2015), part of the MIT Software Studies series, and is broadly mirrored within other work constituting the fields of media archaeology, platform theory, and format theory. ${ }^{4}$ Miller and Horst's definition of the digital also is in tension with the broad approach taken within Actor Network Theory and Science and Technology Studies, which see the social and technological as co-constructed or produced within broader socio-technical networks or assemblages. Today, Digital Anthropology at UCL draws from all of these trajectories - with research focused on the anthropology of data, the materiality of the digital, the infrastructural quality of digital networks, and the politics of digital forms as evidential categories, as well as the communicative and expressive capacities of networked digital communication (see Walford, Coupaye, and Knox's contributions in Chapters 15, 4, and 8). ${ }^{5}$ However, the peculiar location of digital anthropology within broader debates about material culture continue to both intrigue and confuse students, forging ongoing debates about the relationships between form and content, scale of analysis, and location of criticality within digital projects. These questions play out most explicitly in the emerging disjuncture between a critical digital anthropology, and the formulation and representation of research through digital ethnography.

\section{From writing culture to digital ethnography}

As the material turn broadly explores the role that objects play in the constitution of social worlds, sometimes even from the perspective of objects themselves, it is interesting how little critical thinking about ethnography within anthropology connects to the emergence of digital ethnography, and the elaboration of visual and other social or 'inventive methods' (Pink et al. 2016; Lury and Wakeford 2012). The set of reflexive explorations of anthropological representation precipitated by the publication of Writing Culture (Clifford and Marcus 1986) explored the nature of anthropology as a representational practice and challenged anthropology's claims to objectivity by situating and deconstructing the production of ethnographic texts, and later turning attention to visual practices of film and photography. For some reason, this critique is rarely discussed in relation to the form of digital ethnography, even as MCS highlights that representational technologies have the power to alter and affect that which they are trying to capture (see Marres et al. 2018: 24). With the exception of a few key examples - such as the theatrical, exhibitionary, and media lab work of Bruno Latour (e.g. Latour 2005; Venturini et al. 2017 or some of the work of the Harvard Sensory Ethnography Lab (e.g. Castaing-Taylor and Paravel's cross-over 2014 film Leviathan) - multi-media anthropology remains on the margins of the discipline and the digital remains largely unpacked in terms of its representational conventions - even as digital tools for anthropological research proliferate.

Clifford's genealogy for Writing Culture (Clifford 1988) drew on anthropology's links to twentieth-century modernist social theory, in which 
representational forms, such as writing and photography, were linked to other aesthetic avant-gardes in art and literature. Today, 'inventive methods' (Lury and Wakeford 2012) are also positioned at the heart of experimental and creative avant-gardes in the social sciences. Yet the emergence of digital ethnography increasingly draws not just on bespoke or artisinal technical projects (often funded for the short term by research grants), but by necessity on more commonplace digital forms: blogs, social media, databases, and algorithms, many of which have been developed primarily to produce value for corporate shareholders through the production of data related to consumer preference, ultimately to generate revenue through advertising. What then are the implications of these generic forms on the structure as well as on the content of our work as digital anthropologists? If, as Lupton (2015) observes, digital ethnography is simultaneously a form of research, analysis, and criticism, what effects does the black-boxed political economy of social media and its accompanying ingrained culture of obsolescence have on our work?

\section{A case in point: learning from social media in the field}

Digital anthropology is, for me at least, a site of struggle between totalising views of the social as they are constituted within anthropology, and as they are constituted through increasingly generic digital media. In the rest of this chapter, I use as a case study an experimental student project at UCL designed to explore the analytic limits of social media and to highlight how the methods and perspectives of material culture might enable a greater reflexivity within digital projects. By exploring the successes and failures of a student project, I want to briefly look at the ways in which understanding digital media as method not only provides an avenue for forms of social and participatory methodologies but, perhaps more importantly, provides a place for discussion of the relations between form and content, and between theory and ethnography. This is less a 'how-to' primer for utilising social media in anthropological research and more an exploration of how much work we need to do in order to understand the interpenetration of social media and digital methods within anthropology.

The project in question was built around the use of ubiquitous social media platforms. As will be seen, unlike other research projects focused on social media that present social media as an enduring part of people's social worlds (e.g. Miller et al. 2016), the ephemerality, contingency, and fallibility of these projects and their status as partial successes and partial representations, demonstrate some of the false dreams of digital and ethnographic holism as they are packaged up in alternative research environments. The project illustrates a dissonance in the ways in which it is often assumed that social networks map onto digital networks, and it unpacks the nature of digital participation and co-production as well as contributes to the un-black-boxing of technical expertise and skill around the use of everyday platforms that have become 
so ubiquitous that they are considered unskilled or banal by their users (see Geismar and Mohns 2011). Finally, observing the ways in which social media is transformed into method enables us to place digital practices and methods in specific cultural, and local, context.

\section{Social media as community?}

Our methodological exploration of social media was undertaken in collaboration with Dr. Rebecca Ross (University of the Arts: Central St Martins) and students from the 2013-2014 cohorts in the graduate degree programs of UCL Digital Anthropology and CSM Graphic Design and Communication. We proposed to link our students to two community associations in London in order to explore the capacity of social media to build community in particular neighbourhoods, with the intention that our students would 'hack' social media and push it to its limits. The aim of the project was to explore generic, popular social media platforms focusing on the free platforms that were the most popular at that time - YouTube, Twitter, Facebook, and Instagram (one group also worked with a fitnessspecific social networking platform) - doing so in a way that challenged the conventional imaginaries of 'social networking,' using the trope of hacking (as a form of non-normative interventionist participatory methods of active engagement with form) to investigate our definitions of the social, of the network, of communication, sharing, neighbourhood, friendship, participation, authorship, ownership of platforms and content, and so forth. Our brief to the students made it very clear that there are multiple perspectives on social media, the stakes of which can vary across projects and fields of enquiry. We wanted them to be able to critically engage with social media and its conventions in the same way as they had been trained to critically unpack other kinds of media (e.g. documentary photography and film, ethnographic texts).

Our students teamed up into groups of four, and we partnered each group with two community associations in the London Borough of Camden. Working together, they determined the needs and expectations of the association and then brainstormed how to set up a social-media network for them to use over the summer of 2014. The aim was to co-produce with community members something that was at once productive to the community associations, creatively considered, and critically engaged with contemporary social media.

Community associations occupy a liminal place in the landscape of public culture in the UK. Over the past twenty years, community centres have largely shifted from being social or public services to independent, selfgoverning charities, as part of a broader scaling back of national support for local government and civil society. While community associations, as they are now called, are funded in part through the local authority, this is often with short-term or block grants, and they are expected to gain some kind 
of financial autonomy. This money is increasingly competitive and hard to access, and direct funding from government has been cut radically over the past ten years by the Conservative-led national government. Associations are expected to fundraise through social enterprise and by making connections to local businesses. It became clear throughout our project that the community associations we worked with saw our project as a way to participate within a broader agenda in the UK to provide social welfare and community services online. It was also clear they were very aware of the role of digital technologies in implementing the conditions of austerity in regards to public services, where online services were understood as both liberating and democratising, and as a way to transfer responsibility and activity away from state welfare services.

Here, I focus on what happened at the Castlehaven Community Association (CCA) (www.castlehaven.org.uk), an independent charity set on four acres in Camden Town just opposite the cluster of Camden Markets. The railway viaduct divides the neighbourhood, with gentrified railway cottages that are home to affluent professionals and the popular markets on one side and, on the other, a large cluster of social housing estates. Within these estates, despite the economic vibrancy of the market, unemployment is a significant issue. Association CEO at the time, Eleanor Botwright, observed that, in the face of little opportunity, there has been a growing sense of apathy from the younger members of the community.

CCA in 2014 had a garden, playground, outdoor sports ground and two main buildings, as well as managing a large tenant's association hall on the nearby public housing estate. The Haven housed an Internet radio station, recording studio, dance studio with piano, and well-equipped meeting room used during the day as a space for Age Activity (for older people), and in the evening and school holidays as a youth centre. Another building contained staff offices, a large meeting room used as a drop-in for under-fives on Tuesday, Wednesday, and Thursday mornings, and a meeting/activity space offering learning and social opportunities for Age Activity members. The CCA recognises that their spaces are intergenerational and has increasingly built on that in its programming. Many activities are developed in reference to mass media, for instance building on the popularity of reality television shows such as Big Brother, X-Factor, and The Voice, to develop youth activities.

We went into these projects with a series of issues to explore, clustered around the primary question, 'What does social media really want?', drawing inspiration from W.J.T. Mitchell's question 'What do pictures "really" want?' (1996). We were interested to explore how much community engagement we could establish through social media. We also wanted to explore whether we could see social media as being part of a neighbourhood, and whether social media could in fact be a neighbourhood. The students' own activities showed us what we can and cannot do with social media, how much social media foreclosed certain kinds of participation, and helped us understand the limitations of the platforms as well as their affordances. 


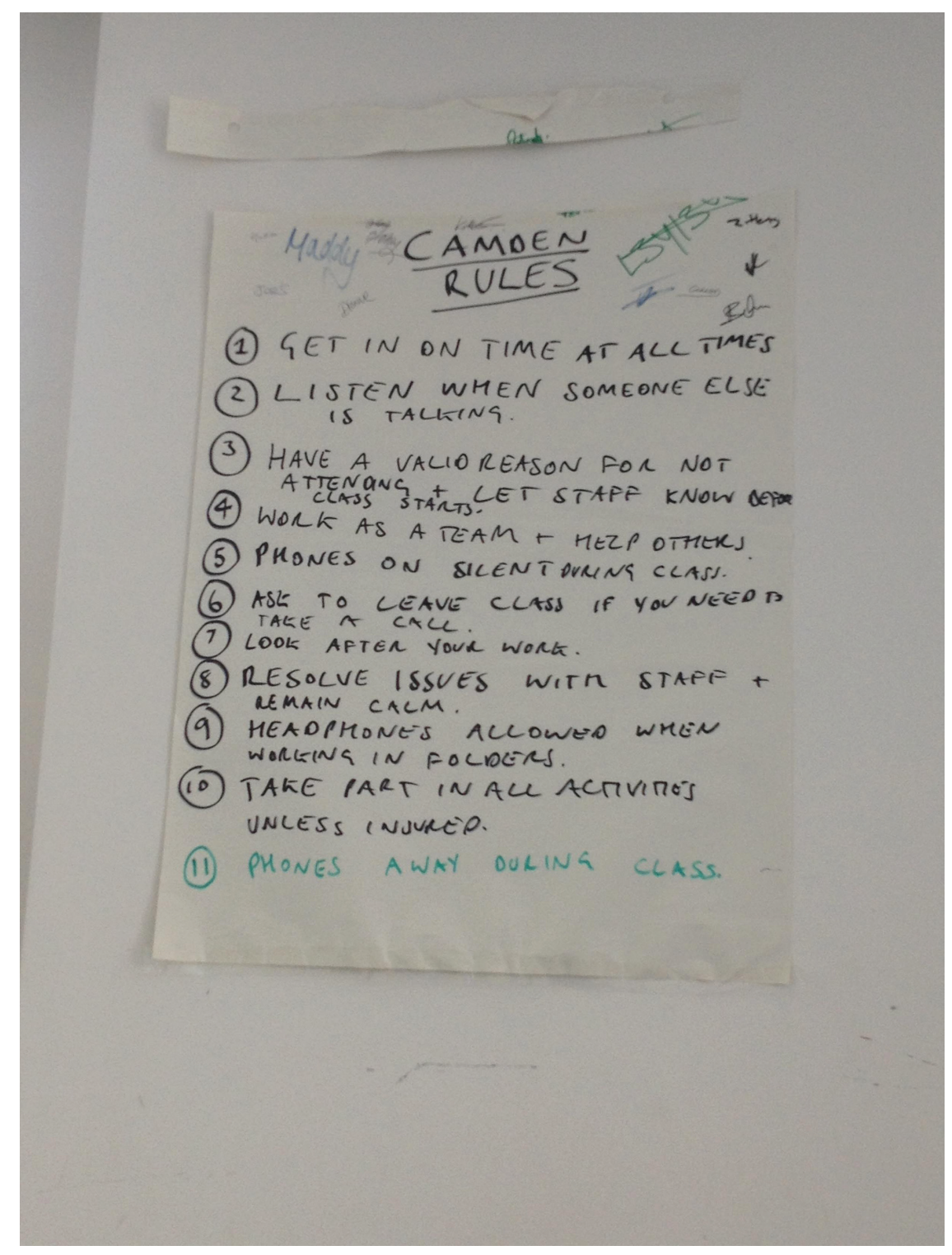

Figure 6.1 'Camden Rules' posted at CCA.

Source: Photo by author.

\section{Camden Rules}

One of the first things we noticed during our first visit in 2014, was a list of house rules in the second-floor club house room of the CCA, written by the 
Youth Coordinator. The rules aimed to structure the engagement of teenagers in weekend and after-school activities, including the production of radio shows, Big Brother style sleepovers, DJ'ing, and open-mike competitions. Rule five stipulates that phones should be on silent during class, rule nine decrees that headphones can only be worn when working in 'folders,' and rule ten states that everyone should participate in everything unless incapacitated. There is an appended, eleventh rule, written in a different colour pen, which admonishes students to put their phones away. The organic development of the house rules reflects the struggles that the CCA is facing to maintain the attention of its networked teens.

We had challenged our students to leverage social media to make people aware of the activities and programmes of the community centre, to connect the different groups on each side of the track and open up more opportunities for lower-income groups to make people aware that the organisation is not part of Camden Council, but an independent charity responsible for its own income generation to develop social enterprise opportunities. The student project focused on developing the recognition that social media is already part of the community association. Rather than developing rules to limit the use of mobiles, the students developed strategies for inclusion through social media. The project focused on drawing attention to the creative aspects of social media and on social media as a form of attention rather than distraction. As the student team wrote:

Given that one of CCA's objective[s] is to drive more youth to their community centre we've suggested they adopt a 'noisier' approach to Instagram and Twitter. The youth at CCA loved the idea of having and sharing pictures from CCA activities on Instagram. This takes both objectives hand in hand: increasing visibility and engaging youth at the community centre to drive more youth to the community centre. What we were concerned about was that the youth who use CCA's resources, especially the recording studio do tweet about being there and what they do there, but this is not picked up by CCA and promoted further. What we'd like to see CCA to do over the next few months is better integrate and showcase what is happening in the studios, offline, in a visual manner online by sharing and engaging with the youth on social media. CCA's rules seem to suppress sharing as opposed to encourage it, so we've drafted a set of new rules for CCA's which encourages sharing on social media and also asks them to tag CCA and use the specific \#makenoise hashtag to promote CCA as much as possible for the youth as well as other members and stakeholders in the community.

The students redeveloped the classroom rules to make space for social media, encouraging the students to use social media as a form of engagement rather than as distraction, creating their own set of rules of engagement that incorporated rather than resisted social media. It became virtually impossible 
to critically engage with the form of social media itself, beyond the genres and forms that it afforded. Social media became both the start and end point of the project, the form and the subject, the primary definition of social engagement and the record of it.

One of the issues this project highlighted was the difficulty students had in developing critical toolkits around social media platforms, partly because of the blurring of boundaries that takes place when undertaking research on social media, while using social media as both a tool and as the form of a project. Despite our intentions to experiment with these ubiquitous platforms and to complicate the ways in which social media is mapped onto community, the default mode of operation for both students and community association leaders was to assume that social media is predominantly a form and method of visibility, especially useful in the competitive market-led environment that governs local government and community life in London today. The leaders of Castlehaven saw no disconnect between their desire to expand their social media presence and their desire to disconnect their teenage users. For them, social media was in the first instance a broadcast medium to afford new recognisable forms of visibility (and attendant forms of value that this visibility produces) for the Centre's activities to a wider constituency.

Our students, on the other hand, were being trained in a shared analytic between graphic design/communication and digital anthropology that explored how social media platforms might instantiate, and mirror, different kinds of sociality. However, it quickly became clear during the project that they also brought to the table a number of contextual or vernacular assumptions about social media. These assumptions raise a question regarding the neutrality of these platforms or whether their functionality has been designed with particular uses in mind, to the extent that they prefigure and pre-program our own capacities as users.

The anthropology of social media is still in its infancy, bringing together a number of different strands from within anthropology. One brings together classic interests in kinship, relationship building, and other forms of sociality to these new technologically mediated networked platforms (e.g. Boyd 2015; Baym 2010; Miller and Slater 2000). Another connects the longer history of media anthropology to unpack these platforms as mediating forms in and of themselves. This work emphasises the contexts of infrastructure and governance (Larkin 2013; Malaby 2009), the social norms that structure online interaction in platforms such as games and virtual worlds (e.g. Nardi 2015; Boellstorff 2008), and cell phones (e.g. Horst and Miller 2006), and the social conventions through which people inhabit these digital environments (Gershon 2010). Anthropologists disagree regarding the ways in which social media may be understood to either map onto social life or produce an inauthentic or alternative vision of the social (see Costa et al. 2016 versus Couldry and Van Dijk 2015). Most recently, as our awareness of how social media platforms function grows, there is a growing anthropology of algorithms and other digital forms that construct a socio-technical 
perspective on the ways in which social media mirrors or represents reality (e.g. Seaver 2019).

Within the teaching rooms of UCL and CSM we are relatively free to train our eyes away from the more dystopian aspects of social media: the corporatisation of social media and its role as a large-scale industry. Despite our intensive engagements with creative practices and grass-roots uses, for many people social media represents opportunities to convert strong or weak ties into valuable connections, to understand the self as partial data, and to participate in the processes of their own surveillance and commoditisation. ${ }^{6}$ The anthropology of social media, then, seemed to have very little capacity to surprise us. Rather, like a hall of mirrors, one keeps seeing the same forms over and over again. Likes, retweets, and portfolios of friends and followers, like many kinds of cultural capital, can be understood by academics to fetishise and alienate the self in their conversion of personal data into financial capital. Even though many users within social media networks may not be directly engaging with critiques of the financialisation of the self, there is also a general awareness of many platforms as being vehicles for the production of value or using transactions to benefit themselves through the production of a networked form of visibility, that is, promoting their careers on LinkedIn, and creating a branded self (Gershon 2017). It is also true that, as well as importing the value structure of marketing and consumer capitalism into their logic, these instrumental socialities also refract social theories developed within the social sciences to produce what Kelty has termed a 'recursive public' (2008) in the specific terms of social media platforms.

\section{Conclusion: social media imaginaries, failing methods, and the limits of objects}

By paying close attention to the imaginaries of social media that emerged within our supposedly experimental project, we were able to understand more fully the assumptions that are developing within both communication design and digital anthropology about social media, as well as within the London Borough of Camden's community associations, who are struggling to keep afloat in the wake of massive budgetary cuts from local and national government. The project showed us how social media is simultaneously an object of analysis and a method through which people use their connections to other people to produce value by making these connections visible. By utilising these forms within our own methods for digital anthropology, are we uncritically constituting a new form of instrumentalism? And if so, what role does MCS play within this?

Marres et al. argue that 'social life is not something that simply exists out there, but [it] is made: the very existence of social life depends on specific practices of display, representation, accounting and enactment' (2018: 19). Their notion of 'inventing the social' recognises the creative work that underpins social research methods, and the performativity of research 
methods in which 'to experiment [means], first and foremost, [...] to intervene in social life, not necessarily with an instrumental goal in mind but to highlight social formations' (2018: 29). Here, I have focused on a particular project, in which students and community members tried to make sense of social media in order to explore the interrelationships between some of the insights of MCS and the new methodologies that are emerging within the domain of digital anthropology. The stalemate of method - in which digital ethnography has become a marketable skill, an index of the qualitative, used strategically to produce value within non-academic research contexts - depends on the complex materiality of the digital, which blurs the boundary between form and content, structure and practice, theory and method. I have also posited a divide between anthropological accounts of the social that emerge from within social media, and those that are understood to exist outside - or a priori to - social media. Marres and Gerlitz argue that social media is a place within which people experiment with the social (2018: 253). What we found in our classroom project is that the social was increasingly constrained by the format of social media. While this is a fundamental tension for the material turn - whose theoretical drivers rest on the question regarding the role of form or materiality in constituting sociality and meaning - the tension between these world views is also pivotal to digital ethnography and other digital methods (see Knox, Chapter 8).

Zuboff argues that what she terms 'the age of surveillance capitalism' marks the rise of what she describes as 'instrumentarian power' (2018: passim) whereby digital technologies are used to shape and mould people's activities, extracting surplus value at every moment in which human activity can be converted into data - an economics and politics presaged on a kind of extreme behaviourism. Within this top-down, nihilistic view of technology, digital anthropology, and most particularly the contribution of ethnographic method, is often presented as a palliative - helping us to understand the experience of everyday life within these technical platforms, and sometimes arguing for greater local or grassroots agency than can often be imagined within perspectives that look down from afar. Given these stakes, I propose we follow Alan Liu's call to 'use the tools, paradigms, and concepts of digital technologies to help rethink the idea of instrumentality' itself (2012). For Liu, this involves 'thinking critically about metadata (and everything else related to digital technologies) in a way that scales into thinking critically about the power, finance, and other governance protocols of the world' (ibid.). This requires us to return to anthropology as critique, not just description, and to unpack the epistemologies that are increasingly hardwired into our methods as they move into digital platforms. These approaches to digital media both extend and complicate the various turns epitomised by today's MCS. Ultimately, it is helpful to remind us of the reactionary qualities of MCS, that it is the return to persistent questions about the entanglements of structure and practice, production and consumption, materiality and sociality that enable us to constitute a true anthropology of the digital as well as a digital anthropology. 


\section{Acknowledgements}

Thanks to Rebecca Ross, Eleanor Botright, and our students (2013-2014) for the project that is at the heart of this chapter, and to Rebecca for critical engagement and contributing to the writing about community associations (all mistakes however are my entirely my own). Thanks also to Daniel Miller, Ludovic Coupaye, Antonia Walford, Shireen Walton, and Timothy Carroll for critical feedback on many drafts.

\section{Notes}

1 Anthropology, like other disciplines, has a long history of being 'put to use' or applied, from the role the discipline has played in supporting colonial government (Asad 1973), through to the embedding of anthropologists and their work in the military (Price 2011). A ubiquitous political economy of audit and value within teaching, research, and now increasingly engagement with the outside world (variously referred to in the UK as impact, knowledge transfer, knowledge exchange, and public engagement) increasingly structures the values of the modern British university. It is no coincidence that some of the most prominent theorists of MCS have also turned their attention to audit cultures that explore the ways in which new regimes of value produce the need for new knowledge and information economies and vice versa (see Miller 2005a; Strathern 2003). Miller's initial work on audit was part of a bigger project focused on 'virtualism' which linked a number of shifts in the production of value: towards audit rather than information, consumers (rather than commodities), and management as a particular form of labour borne out of the deliberate shift towards concepts rather than objects in late twentieth century political economies (see Carrier and Miller 1998).

2 In many ways, this approach mirrors contemporary debates within media studies and media anthropology in which media (or digital) is articulated as a 'total social fact' (taken from Mauss' articulation of 'the gift' as a total social fact, 2002[1925, 1976]). Miller works with a definition of the digital as 'all that can be ultimately reduced to binary code, but which produces a further proliferation of particularity and difference' (Miller and Horst 2012:3).

3 www.ucl.ac.uk/why-we-post/discoveries/5-people-who-use-social-media-whocreate-it-not-the-developers-of-platforms, last accessed 31 May 2019.

4 Indicative references are in relation to media archaeology (Parikka 2012b); digital humanities (Lui 2012, Risam 2019); software studies (Manovich 2013); platform theory (Dourish 2016); format theory (Sterne 2012) and new materialism (Henning 2007).

5 More widely, see Knox and Nafus 2018; Walford 2015; Adriaans 2019; Miller and Sinanan 2013; Geismar 2018.

6 See for example this report in the Guardian newspaper, recounting how TikTok users are 'used to being tracked' and are 'unfazed' by corporate data collection: www. theguardian.com/technology/2020/aug/05/tiktok-gen-z-millennials-data-privacytrump-china (last accessed 21, 08, 2020). 